\title{
Relation between Exponential Stability and Input-to-State Stability of Time-Delay Systems
}

\author{
Nima Yeganefar, Michel Dambrine and Nader Yeganefar
}

\begin{abstract}
The main contribution of this paper is to establish a link between the exponential stability of an unforced system and the Input-to-State Stability (ISS) via the LyapunovKrasovskii methodology. A new theorem is provided, which proves that an unforced system whose trivial solution is exponentially stable is input-to-state stable if submitted to a perturbation which can be of an arbitrary size.

Keywords: Input-to-State Stability, Exponential Stability, Nonlinear Time-Delay Systems, Lyapunov-Krasovskii Theorem.
\end{abstract}

\section{INTRODUCTION}

For non-delayed systems, the Input-to-State Stability (ISS) property has been widely studied and its efficiency has been proved in practical applications such as networked control and robot manipulators (see [1] and the references therein). ISS implies not only that the unperturbed system is asymptotically stable in the Lyapunov sense but also that its behavior remains bounded when its inputs (eg. exogenous perturbations) are bounded. Recently, some authors have attempted to address the lack of results regarding time-delay systems (see [10] and the references therein). Specifically, in this short communication, we will exhibit a link between exponential stability and the ISS property. Exponential stability has proved its efficiency in networked control (see eg. [6]). However, the influence of disturbances on the solutions behaviour have to be more deeply analyzed from both a qualitative and a quantitative point of view. For networked control systems, a first work in this direction is the paper [7] by Polushin and Marquez relying on Teel's results ([3]), which may be somewhat conservative due to the use of Razumikhin-Lyapunov functions. We show in this paper a link between ISS and exponential stability for a large class of systems which means an easy way of checking ISS for these systems.

\section{Notations:}

For $y \in \mathrm{R}^{n},\|y\|$ denotes the Euclidean norm of the vector $y$; for a measurable function $x(t),|x|_{\infty}=\sup \{\|x(t)\|, t \geq 0\}$ is the (essential) supremum norm. For a given $\tau>0, \mathscr{C}$ denotes the space of continuous functions mapping the interval $[-\tau, 0]$ into $\mathrm{R}^{n}$ and for $\varphi \in \mathscr{C},\|\varphi\|_{c}=\sup _{-\tau \leq \theta \leq 0}\|\varphi(\theta)\|$. $\mathscr{C}_{H}$ will denote the set of $\varphi \in \mathscr{C}$ such that $\|\varphi\|_{c} \leq H$. As

\footnotetext{
Nima Yeganefar is with LAGIS UMR CNRS 8146, Ecole Centrale de Lille, 59651 Villeneuve-d'Ascq, France nima.yeganefardec-lille.Fr

M. Dambrine is with LAMIH UMR CNRS 8530, Universit de Valenciennes, 59313, Valenciennes, France michel.dambrineduniv-valenciennes. fr

Nader Yeganefar is with the Department of Mathematics, LATP UMR 6632, 13453 Marseille, France nader.yeganefarecmi.univ-mrs.fr
}

usual, the symbol $x_{t}$ will denote the element of $\mathscr{C}$ defined by $x_{t}(\theta)=x(t+\theta),-\tau \leq \theta \leq 0$. The reader can find the definitions of class $\mathscr{K}, \mathscr{K}_{\infty}$ and $\mathscr{K} \mathscr{L}$ in [8, p.144]. Finally $D^{+} V\left(t, x_{t}\right)$ is the derivative of a functional $V$ along the trajectory of the studied system (see for instance [9]).

\section{EXPONENTIAL STABILITY OF UNFORCED SYSTEMS}

We will consider the exponential stability problem for the following equation in $\mathrm{R}^{n}$ with bounded delay $\tau>0$ :

$$
\left\{\begin{array}{c}
\dot{x}(t)=f\left(x_{t}\right), \quad t \geq t_{0}, \quad f(0)=0 ; \\
x_{t_{0}}=\psi,
\end{array}\right.
$$

where $\psi \in \mathscr{C}_{H}$, and $f: \mathscr{C}_{H} \rightarrow \mathrm{R}^{n}$ is continuous, locally Lipschitz with constant $L>0$. In [10], an interesting upperright hand derivative is presented which will be helpful in proving our main theorem.

Given a continuous functional $V: \mathscr{C} \rightarrow \mathrm{R}^{+}$, the upper righthand derivative $D_{*}^{+} V$ of the functional $V$ is given by (see [11])

$$
D_{*}^{+} V(\varphi)=\limsup _{\Delta t \rightarrow 0^{+}} \frac{1}{\Delta t}\left(V\left(\varphi_{\Delta t, u}^{*}\right)-V(\varphi)\right),
$$

where $\varphi_{\Delta t, u}^{*} \in \mathscr{C}$ is given by

$$
\varphi_{\Delta t, u}^{*}(s)=\left\{\begin{array}{l}
\varphi(s+\Delta t), \quad s \in[-\tau,-\Delta t] \\
\varphi(0)+f(\varphi)(\Delta t+s), \quad s \in[-\Delta t, 0] .
\end{array}\right.
$$

Note that this definition is quite different from the one presented in the introduction. But if the function $f$ is continuous, the definitions are equivalent when the functional $V$ is locally Lipschitz ([11] and [12]); this will be our case.

The next theorem, proved in [13, Lemma 33.1], will play an important role in the proof of our main theorem.

Theorem 2.1: If there exist two strictly positive constants $A$ and $B$ such that for every $t \geq t_{0}$ and $\psi \in \mathscr{C}_{H}$ the solution $x\left(t ; t_{0}, \psi\right)$ of system (1) satisfies the condition

$$
\left\|x_{t}\left(t_{0}, \psi\right)\right\|_{c} \leq A\|\psi\|_{c} e^{-B\left(t-t_{0}\right)}, \quad t \geq t_{0},
$$

then there exists a continuous functional $V(\varphi)$ defined on $\mathscr{C}_{H}$ which satisfies the following conditions:

$$
\begin{aligned}
& C_{1}\|\varphi\|_{c} \leq V(\varphi) \leq C_{2}\|\varphi\|_{c}, \\
& D^{+} V(\varphi) \leq-C_{3}\|\varphi\|_{c}, \\
& \|V(\varphi)-V(\xi)\|_{c} \leq C_{4}\|\varphi-\xi\|_{c},
\end{aligned}
$$

where $C_{i}$ are some positive constants, $\varphi$ and $\xi$ are arbitrary functions from $\mathscr{C}_{H}$. 


\section{MAIN RESULT}

As previously stated, a definition of input-to-state stability for time-delay systems has been given in [3] and a useful characterization has been presented in [4]. For the reader's convenience, we reproduce here the definition of ISS. Consider the system

$$
\dot{x}(t)=f\left(x_{t}, u\right), \quad t \geq t_{0},
$$

where $f$ is a continuous functional defined on $\mathscr{C} \times \mathrm{R}^{m}$. The input $u$ is a piecewise continuous bounded function of $t$ for all $t \geq t_{0}$. Consider also the unforced system

$$
\dot{x}(t)=f\left(x_{t}, 0\right), \quad t \geq t_{0},
$$

Definition 3.1: The system (8) is said to be input-to-state stable if there exist a class $\mathscr{K} \mathscr{L}$ function $\beta$ and a class $\mathscr{K}_{\infty}$ function $\gamma$ such that, for any initial state $\psi$ and any locally essentially bounded input $u$, the solution $x_{t}\left(t_{0}, \psi\right)$ exists for all $t \geq t_{0}$ and satisfies

$$
\left\|x\left(t ; t_{0}, \psi\right)\right\| \leq \beta\left(\|\psi\|_{c}, t-t_{0}\right)+\gamma\left(|u|_{\infty}\right) .
$$

Definition 3.2: A continuous functional $V: \mathscr{C} \rightarrow \mathrm{R}^{+}$is called an ISS-Lyapunov functional for system (8) if there exist $\mathscr{K}_{\infty}$-functions $a, b$, and $\mathscr{K}$-functions $\chi$ and $\alpha_{3}$ such that

1) $a(\|\varphi(0)\|) \leq V(\varphi) \leq b\left(\|\varphi\|_{c}\right)$

2) $D^{+} V(\varphi, u)_{(8)} \leq-\alpha_{3}\left(\|\varphi\|_{c}\right), \quad \forall\|\varphi\|_{c} \geq \chi(|u|)>0$

The following result is the main contribution of [4]:

Theorem 3.3: If system (8) admits an ISS-LyapunovKrasovskii functional then it is ISS.

Our main theorem can now be stated as follows:

Theorem 3.4: Suppose $f\left(t, x_{t}, u\right)$ is continuously differentiable and globally Lipschitz in $\left(x_{t}, u\right)$, uniformly in $t$. If the unforced system (9) has a globally exponentially stable equilibrium point at the origin $x=0$, then the perturbed system (8) is input-to-state stable.

Proof: We view system (8) as a perturbation of the unforced system (9). The converse Lyapunov theorem 2.1 shows that the unforced system (9) has a LyapunovKrasovskii functional $V(\varphi)$ that satisfies the inequalities (57). Please note that $V$ is locally Lipschitz (7). Computing the upper right-hand derivative of the functional $V$ as in (2) along the trajectories of the forced system (8), we get:

$$
\begin{aligned}
& D_{*}^{+} V(\varphi, u)=\limsup _{\Delta t \rightarrow 0} \frac{1}{\Delta t}\left(V\left(\varphi_{\Delta t, u}^{*}\right)-V(\varphi)\right) \\
& =\limsup _{\Delta t \rightarrow 0} \frac{1}{\Delta t}\left(V\left(\varphi_{\Delta t, u}^{*}\right)-V\left(\varphi_{\Delta t, 0}^{*}\right)-V(\varphi)+V\left(\varphi_{\Delta t, 0}^{*}\right)\right) \\
& \leq-C_{3}\|\varphi\|_{c}+\limsup _{\Delta t \rightarrow 0} \frac{1}{\Delta t}\left(V\left(\varphi_{\Delta t, u}^{*}\right)-V\left(\varphi_{\Delta t, 0}^{*}\right)\right) .
\end{aligned}
$$

Observe the following inequalities:

$$
\begin{aligned}
\| V\left(\varphi_{\Delta t, u}^{*}\right)- & V\left(\varphi_{\Delta t, 0}^{*}\right)\left\|_{c} \leq C_{4}\right\| \varphi_{\Delta t, u}^{*}-\varphi_{\Delta t, 0}^{*} \|_{c} \\
& =C_{4} \sup _{s \in[-\tau, 0]}\left\|\varphi_{\Delta t, u}^{*}(s)-\varphi_{\Delta t, 0}^{*}(0)\right\| \\
& \leq C_{4} \sup _{s \in[-\Delta t, 0]}|\Delta t+s|\|f(\varphi, u)-f(\varphi, 0)\| \\
& \leq C_{4}|\Delta t| \ell\|u\| \leq C_{4}|\Delta t| \ell|u|_{\infty}
\end{aligned}
$$

due to the uniform global Lipschitz property of $f$. Hence

$$
D_{\star}^{+} V(\varphi, u) \leq-C_{3}\|\varphi\|_{c}+C_{4} L|u|_{\infty} .
$$

As $V$ is locally Lipschitz, $D_{\star}^{+} V=D^{+} V$ (see part II). It can be easily shown that this functional is an ISS-LyapunovKrasovskii functional as defined above. The idea is to use the term $-C_{3}\|\varphi\|_{c}$ to dominate $C_{4} L|u|_{\infty}$ for large $\|\varphi\|_{c}$ :

$$
D^{+} V(\varphi, u) \leq-C_{3}(1-\theta)\|\varphi\|_{c}, \quad \forall\|\varphi\|_{c} \geq \frac{C_{4} L|u|_{\infty}}{C_{3} \theta},
$$

for all $(\varphi, u)$ and $0<\theta<1$ and from theorem 3.3 we conclude that the system is input-to-state stable.

\section{ACKNOWLEDGEMENT}

The first author would like to thank Darian Meacham, Institute of Philosophy K.U. Leuven, for the english corrections of this article.

\section{CONCLUSIONS}

Let us sum up the main lines of this paper. We first highlighted the importance of the ISS theory, recently adapted for nonlinear time-delay systems, as one of the best candidates for analyzing actual problems such as networked control, hybrid or even quantized systems.

In this context, we established a new connection between ISS and exponential stability. We showed that if the unforced system is exponentially stable, the forced system is ISS, keeping in mind that the considered input is an exogenous perturbation.

\section{REFERENCES}

[1] E. Sontag, "the ISS philosophy as a unifuing framework for stabilitylike behavior," Nonlinear Control in the Year 2000 (Volume 2), pp. 443-468, 2000.

[2] — "Smooth stabilization implies coprime factorization," IEEE Trans. Aut. Control, vol. vol. 34(4), pp. 435-443, 1989.

[3] A. Teel, "Connections between Razumikhin-type theorems and the ISS nonlinear small gain theorem," IEEE Trans. Aut. Control, vol. vol. 43(7), pp. 960-964, 1998.

[4] P. Pepe and Z.-P. Jiang, "A Lyapunov-Krasovskii methodology for ISS of time-delay systems," in Proc. Of the 44th IEEE CDC and ECC, Seville, Spain, 2005.

[5] D. Liberzon, "Quantization, time delays, and nonlinear stabilization," IEEE Trans. Aut. Control.

[6] A. Seuret, M. Dambrine, and J.-P. Richard, "Robust exponential stabilization for systems with time-varying delays," in TDS'04, IFAC Workshop Time-Delay Systems, Leuven, Belgium, Sept. 2004.

[7] I. Polushin and H. Marquez, "Stabilization of bilaterally controlled teleoperators with communication delay: an ISS approach," Systems and Control Letters, vol. vol. 24, pp. 351-359, 2003.

[8] H. Khalil, Nonlinear Systems, 3rd ed. Englewood Cliffs, NJ: Prentice Hall, 2002.

[9] V. Kolmanovskii and A. Myshkis, Applied Theory of Functional Differential Equations. Kluwer Acad. Pub., 1992.

[10] P. Pepe and Z.-P. Jiang, "A Lyapunov-Krasovskii methodology for ISS and iISS of time-delay systems," Systems \& Control Letters, vol. vol. 55(12), pp. 1006-1014, 2006.

[11] T. Burton, Stability and periodic solutions of ordinary and functional differential equations. Academic Press, Inc., 1985.

[12] T. Yoshizawa, Stability Theory by Liapunov's Second Method. Tokyo: The Mathematical Society of Japan, 1966.

[13] N. Krasovskii, Stability of motion. Stanford University Press., 1963. 